\title{
The Salivary $\beta$-HEX A\% Index as an Excellent Marker of Periodontitis in Smoking Alcohol-Dependent Persons
}

\author{
Napoleon Waszkiewicz, ${ }^{1}$ Beata Zalewska-Szajda, ${ }^{2}$ Sylwia Chojnowska, ${ }^{3}$ \\ Sławomir Dariusz Szajda, ${ }^{4}$ Anna Zalewska, ${ }^{5}$ Beata Konarzewska, ${ }^{1}$ Agata Szulc, ${ }^{1}$ \\ Aleksandra Wojtulewska-Supron, ${ }^{1}$ Alina Kępka, ${ }^{6}$ Małgorzata Knaś, ${ }^{7}$ Jerzy Robert Ladny, ${ }^{4}$ \\ Robert Milewski, ${ }^{8}$ and Krzysztof Zwierz' \\ ${ }^{1}$ Department of Psychiatry, Medical University of Bialystok, Plac Brodowicza 1, 16-070 Choroszcz, Poland \\ ${ }^{2}$ Department of Imaging Diagnostics, Medical University of Białystok, Children Hospital, ul. Waszyngtona 17, 15-274 Białystok, Poland \\ ${ }^{3}$ Medical Institute, College of Computer Science and Business Administration, ul. Akademicka 1, 18-400 Łomża, Poland \\ ${ }^{4}$ Department of Emergency Medicine and Disasters, Medical University of Białystok, ul. Szpitalna 37, 15-295 Białystok, Poland \\ ${ }^{5}$ Department of Pedodontics, Medical University of Bialystok, ul. Waszyngtona 15A, 15-274 Bialystok, Poland \\ ${ }^{6}$ Department of Biochemistry and Experimental Medicine, The Children's Memorial Health Institute, \\ Al. Dzieci Polskich 20, 04-730 Warsaw, Poland \\ ${ }^{7}$ Research Laboratory of Cosmetology, Medical University of Białystok, ul. Akademicka 3, 15-267 Białystok, Poland \\ ${ }^{8}$ Department of Statistics and Medical Informatics, Medical University of Białystok, ul. Szpitalna 37, 15-295 Biatystok, Poland \\ ${ }^{9}$ Medical College of the Universal Education Society, ul. Adama Mickiewicza 59, 18-400 Łomża, Poland \\ Correspondence should be addressed to Napoleon Waszkiewicz; napoleonwas@yahoo.com
}

Received 16 July 2013; Revised 17 September 2013; Accepted 17 September 2013

Academic Editor: George Perry

Copyright (C) 2013 Napoleon Waszkiewicz et al. This is an open access article distributed under the Creative Commons Attribution License, which permits unrestricted use, distribution, and reproduction in any medium, provided the original work is properly cited.

Background. Severe periodontitis leading to tooth loss is found in $5-15 \%$ of most populations worldwide. Aim. The applicability of salivary $\beta$-hexosaminidase ( $\beta$-HEX A\%, percentage of $\beta$-HEX A isoenzyme to total $\beta$-HEX) and $\beta$-HEX B\% $(\beta$-HEX B/ $\beta$ HEX) indexes was investigated as a possible marker of periodontitis. Methods. Thirty three alcohol-dependent smokers (AS) and 32 healthy controls (C) were enrolled in the study. The activity of $\beta$-HEX was measured spectrophotometrically. Results. $\beta$-HEX A\% was significantly higher and $\beta$-HEX B\% was lower in AS than in C group. We found a significant correlation between $\beta$-HEX A\% and gingival index (GI) and an inverse correlation between $\beta$-HEX A\% and salivary flow (SF), in all groups. Salivary $\beta$-HEX A\% index in smoking alcoholics at 0.23 had excellent sensitivity (96\%) and specificity (91\%); the AUC for $\beta$-HEX A\% was high (0.937). There were no correlations between amount/duration-time of alcohol drinking/smoking and $\beta$-HEX A $\%$ or $\beta$-HEX B\%. We found significant correlations between the time period of denture wearing and GI, papilla bleeding index (PBI), and decayed missing filled teeth index (DMFT) and between GI and the amount of smoked cigarettes per day. Conclusion. Bad periodontal state was most likely due to the nicotine dependence. Salivary $\beta$-HEX A\% is a promising excellent marker for the diagnosis of periodontitis.

\section{Introduction}

Periodontium is a group of specialized tissues that surround and support the teeth and maintain them in the maxillary and mandibular bones, and it consists of four principal components: gingiva, periodontal ligament, cementum, and alveolar bone [1]. The inflammatory state of periodontium is called periodontitis, which is one of the most common oral diseases and is characterized by the loss of connective tissue attachment and bone around the teeth in conjunction with the formation of periodontal pockets due to the apical migration of the junctional epithelium (gingival inflammation and alveolar bone resorption) [2]. Periodontitis is generally described as a multifactorial irreversible and cumulative condition, initiated and propagated by bacteria and host factors. Severe periodontitis leading to tooth loss was found in 5-15\% of most 
populations worldwide. It is known that the oral mucosal surface is the home of numerous normal flora microorganisms (more than 500 different bacterial species are able to colonize the oral biofilm), being the portal of entry for the majority of pathogens. It is also known that salivary proteins participate in the protection of oral tissues, as well as upper digestive and respiratory tracts, against a number of microbial pathogens. Therefore, potential periodontal and salivary changes may influence the general health [2-7].

Almost $4 \%$ of global deaths, $4.6 \%$ of diseases and injuries, and $30 \%$ of health care costs are attributed to alcohol abuse [8]. Alcohol drinking is the world's third largest risk factor for disease and disability, whereas in middle-income countries it is the greatest risk [9]. About $2 \%$ of the worldwide population is dependent on alcohol. Up to $90 \%$ of alcohol-dependent patients are reported to smoke cigarettes $[10,11]$.

The association between alcohol addiction and poor dental state, periodontal state, and oral hygiene is widely known [12]. It was also found that persons addicted to alcohol with poor dental status have double concentration of acetaldehyde in the saliva, than those addicted to alcohol and taking care of oral hygiene [13]. In alcohol abusers, reduced salivary flow may lead to inflammatory states of the oral cavity, periodontal diseases, or infections [14]. The association between cigarette smoking and poor oral hygiene is also widely known [12, 15]. Cigarette smoking was observed as the strongest of the modifiable risk factors for periodontal disease, second only to bacterial plaque [16].

Analysis of saliva and gingival crevicular fluid (GCF) may be especially beneficial in the determination of current periodontal status [3]. There is a need to develop the noninvasive, cost-effective, and easy-to-use diagnostic tests and to analyze periodontal diagnosis with identification of these markers for the risk of developing periodontitis, active periodontal disease, to manage patient and response to treatment, to reflect the extent of periodontal destruction, and to predict the future disease progression. So far, some salivary and GCFderived enzyme markers (e.g., elastase, $\beta$-glucuronidase, $\beta$ hexosaminidase, and lysozyme) and nonenzymatic salivary protein markers (e.g., platelet-activating factor, growth factors, fibronectin, lactoferrin, and immunoglobulins) have been identified [3]. However, measuring probing depth and clinical attachment level by calibrated periodontal probe and assessing gingival inflammation by gingival index (GI) and papilla bleeding index (PBI) are still the most reliable and specific clinical periodontal parameters for periodontal diagnosis [2].

Since $\beta$-HEX B\% highly improved the sensitivity of serum $\beta$-HEX, for example, in the diagnosis of alcohol abuse [17], we investigated the salivary index of $\beta$-HEX A\% or $\beta$-HEX B\% to be a useful potential method to increase the salivary $\beta$-HEX sensitivity and specificity, as markers of periodontitis.

\section{Materials and Methods}

2.1. Participants. 65 individuals, participated in the study comprised of 33 alcohol-dependent smoking individuals (9 females and 24 males) (mean age \pm SD; $44 \pm 0.3$ ), recruited from the Alcohol Detoxification Unit of the Psychiatric Hospital in Choroszcz, Poland, and the control group consisted of 32 healthy social drinkers ( 10 females and 22 males) (mean age $41 \pm 7.0$ ) with no history of alcohol abuse or smoking. The chronic alcohol intoxication ranged from 3 to 90 days (mean 23); they drank an average of 232 (100-700) g of alcohol/day. Alcohol-dependent individuals met the criteria for the alcohol and nicotine dependence according to ICD-10 and DSM-IV criteria. The average time of dependence was 13 years for the alcohol and 21 years for the smoking. Alcoholics smoked an average of 18 cigarettes daily.

The study was approved by the Bioethical Committee of the Medical University of Bialystok, Poland. Informed written consent was obtained from all the subjects after explanation of the nature, purpose, and potential risks of the study.

Data and Sample Collection. Material from persons admitted to the Detoxification Unit was collected at the second day of the abstinence period. The subjects were instructed to refrain from smoking, food, and beverages, except water, for 2 hours before saliva collecting. All salivary samples $(3 \mathrm{~mL}$ of resting whole saliva) were collected in plastic tubes placed on ice by spitting method, under standardized conditions [18, 19], between 8:00 am and 9:00 am, to minimize the influence of circadian rhythms. The samples were centrifuged at 3,000 $\times \mathrm{g}$ for 20 minutes at $4^{\circ} \mathrm{C}$, to remove cells and debris. The resulting supernatants were divided into $200 \mu \mathrm{L}$ portions, frozen, and kept at $-80^{\circ} \mathrm{C}$, until analyzed. Salivary flow (SF) was calculated by dividing the volume of saliva by the time of its collection. In all samples, the activity of total $\beta$-HEX, $\beta$-HEX A and $\beta$-HEX $\mathrm{B}$, was assayed in duplicate, and the means of the duplicate results were used as final values.

Gingival status was assessed using gingival index (GI) and papilla bleeding index (PBI). GI scores the marginal and interproximal tissues separately on the basis of 0 to 3 . The criteria are 0 -normal gingiva; 1 -mild inflammation, slight change in color and slight edema but no bleeding on probing; 2-moderate inflammation, redness, edema, and glazing and bleeding on probing; 3-severe inflammation, marked redness and edema and ulceration with a tendency to spontaneous bleeding. The intensity of any bleeding in PBI is recorded as 0 -no bleeding; 1-a single discreet bleeding point; 2-several isolated bleeding points, or a single line of blood appears; 3-the interdental triangle is filled with blood shortly after probing; 4-profuse bleeding occurs after probing; blood flows immediately into the marginal sulcus. Good periodontal health and dental health were interpreted as follows: $<1$ for PBI and GI and $<20$ for DMFT (decayed, missing, or filled teeth). The DMFT index was used to assess the dental state.

2.2. Analytical Methods. Activities of total $\beta$-HEX and its isoenzymes in supernatants of saliva were determined by the colorimetric method [20]. The indexes of $\beta$-HEX A\% and $\beta$-HEX $\mathrm{B} \%$ were calculated by dividing, respectively, the activity of $\beta$-HEX A and $\beta$-HEX $B$ by the total activity of $\beta$-HEX. 
2.3. Statistical Analysis. Statistical analysis was performed with Statistica version 10.0 (Statsoft, Cracow, Poland). The comparisons between groups were made using MannWhitney $U$ test with appropriate adjustment to the critical value of $P$. Descriptive characteristics are presented as means $\pm 1 \mathrm{SD}$, whereas the results of the nonparametric analysis are presented as least square means \pm 1 SEM (standard error of the mean). Spearman's rank correlation coefficient was used to measure the statistical dependence between two variables in alcohol-dependent persons. A receiver operating characteristic (ROC) analysis, calculations of specificity, sensitivity and area under the ROC curve (AUC), and AUC comparisons (for paired samples) were performed using STATISTICA Rapid Deployment of Predictive Models. Statistical significance was assumed at $P<0.05$.

\section{Results}

3.1. Differences between Controls and Smoking Alcoholics. There were no statistical differences in age between controls and smoking alcoholics.

The mean $\beta$-HEX A\% values in C and AS groups were, respectively, $11.5 \pm 19.6$ and $45.5 \pm 16.8$. In AS group, $\beta$ HEX A\% was significantly higher than in $\mathrm{C}$ group $(P<$ $0.001)$. The mean $\beta$-HEX B $\%$ values in C and AS groups were, respectively, $87.9 \pm 19.5$ and $54.8 \pm 17.3$. In AS group, $\beta$-HEX $\mathrm{B} \%$ was significantly lower than that in $\mathrm{C}$ group $(P<0.001)$ (Figure 1).

We did not find any significant correlations between amount/duration-time of alcohol drinking/smoking (or time of alcohol/smoking dependence) and $\beta$-HEX A $\%$ or $\beta$-HEX $\mathrm{B} \%$. There were significant correlations between $\beta$-HEX A\% and GI $(r=0.479, P=0.001)$ and inverse correlations between $\beta$-HEX B\% and DMFT index $(r=-0.309, P=$ $0.038)$ as well as between $\beta$-HEX B\% and GI $(r=-0.433$, $P=0.005)$. We found also a significant inverse correlation between $\beta$-HEX A\% and SF $(r=-0.501, P<0.001)$ and a positive correlation between $\beta$-HEX B\% and SF $(r=0.526$, $P<0.001)$. An inverse correlation was also found between $\beta$-HEX A $\%$ and $\beta$-HEX B\% $(r=-0.987, P<0.001)$.

3.2. Associations between Variables in Control Group. We found significant correlations between GI and PBI $(r=0.840$, $P=0.0000)$, as well as between DMFT index and age $(r=0.448, P=0.021)$. There were significant correlations between $\beta$-HEX B\% and PBI $(r=0.409, P=0.037)$ and GI $(r=0.421, P=0.031)$.

3.3. Associations between Variables in Alcohol-Dependent Persons. We found significant correlations between DMFT index and PBI $(r=0.522, P=0.021)$, the time period of denture wearing $(r=0.463, P=0.022)$, and age $(r=$ $0.448, P=0.021)$. We found also significant correlations between GI and the time period of denture wearing $(r=$ $0.743, P=0.0004)$, DMFT index $(r=0.652, P=0.002)$, PBI $(r=0.721, P=0.0007)$, and the amount of smoked cigarettes per day $(r=0.627, P=0.009)$. There was also a significant correlation between PBI and the time period of denture wearing $(r=0.530, P=0.023)$.

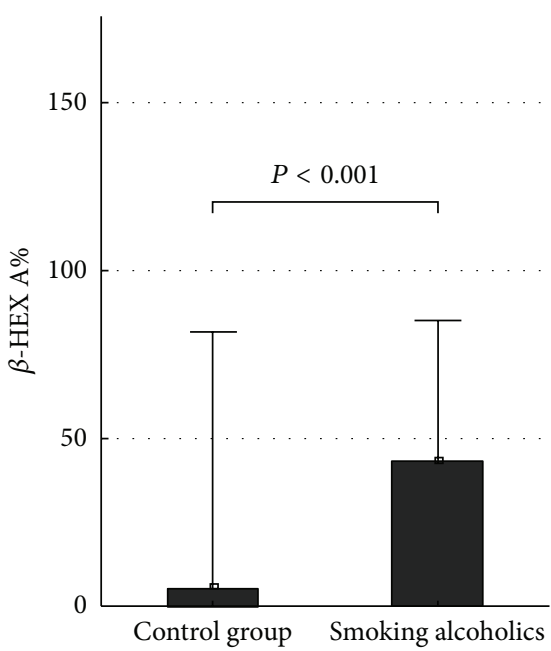

(a)

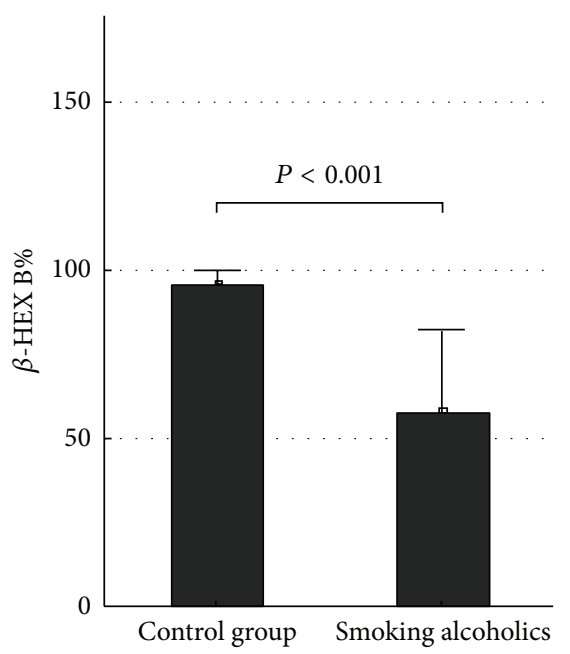

(b)

Figure 1: Comparisons between $\beta$-HEX A\% (a) and $\beta$-HEX B\% (b) values between controls and smoking alcoholics.

3.4. Diagnostic Accuracy of the Salivary Total $\beta$-HEX A\% Index in Smoking Alcohol-Dependent Persons. Obtained by ROC analysis, $\beta$-HEX A\% cut-off values showed that the measurement of $\beta$-HEX A\%, in the saliva of smoking alcoholics, at 0.23 has excellent sensitivity (96\%) and specificity (91\%). The AUC for $\beta$-HEX A\% was of high diagnostic value (0.937 \pm 0.029) (Figure 2).

3.5. Oral Cavity Parameters. The salivary flow (SF) was significantly lower in AS group than in the controls (Table 1). The mean decayed, missing, and filled teeth index (DMFT) had a tendency to be higher, and gingival index (GI) and papilla bleeding index (PBI) were significantly higher in smoking alcoholics (AS) than in controls (C).

\section{Discussion}

During periodontitis state, white blood cells, of which the most troublesome and abundant are polymorphonuclear 


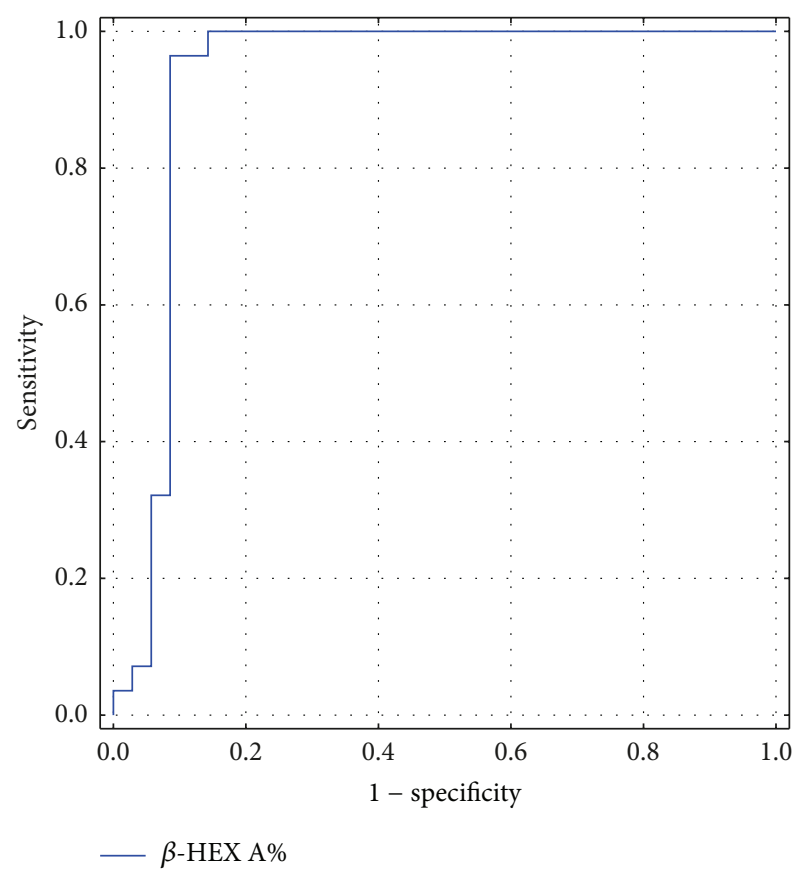

FIGURE 2: Receiver operating characteristic curves for the $\beta$-HEX $\mathrm{A} \%$ index in smoking alcoholics.

TABLE 1: Comparisons of salivary flow (SF), decayed, missing, filled, and teeth index (DMFT), gingival index (GI), and papilla bleeding index (PBI) in controls (C) and smoking alcohol-dependent persons (AS).

\begin{tabular}{lccc}
\hline Variable & C & AS & $P$ value \\
\hline SF $(\mathrm{mL} / \mathrm{min})$ & $0.64 \pm 0.34$ & $0.24 \pm 0.26$ & $<0.001$ \\
DMFT & $18.2 \pm 5.31$ & $21.1 \pm 5.73$ & 0.064 \\
GI & $0.26 \pm 0.45$ & $1.20 \pm 0.82$ & $<0.001$ \\
PBI & $0.38 \pm 0.57$ & $0.90 \pm 0.80$ & 0.007 \\
\hline
\end{tabular}

Values are expressed as mean $\pm \mathrm{SEM} ; P$ value $<0.05$ was considered statistically significant.

$P$ value: $\mathrm{C}$ to AS group.

leukocytes (PMNs), signal for more and more white cells to be recruited to the bacteria site. The congregation of too many PMNs leads to overproduction of noxious agents which are intended to fight the bacteria but overinduce inflammation of healthy tissue and encourage the progression of bone loss and periodontal disease [6]. Neutrophils play a destructive role in the periodontal tissue breakdown process due to high levels of lysosomal enzymes, generation of superoxides, and reactive oxygen species (ROS). In periodontitis, bacterial virulence factors result directly in degradation of host tissues or cause the release of biologic mediators from host tissue cells (proteinases, cytokines, and prostaglandins) that lead to host tissue destruction [3].

Ethanol, which is directly toxic to the mucosa of the oral cavity (including periodontium), mouth, throat, esophagus, and the stomach, diffuses rapidly into saliva and oral tissues and immediately after drinking its salivary concentration is temporarily much higher than that in plasma [21, 22].
After alcohol intake, acetaldehyde is locally formed in the oral cavity oxidation system through oral mucosal alcohol dehydrogenase and oral microflora. As further metabolism of acetaldehyde to acetate via oral bacteria is limited, the level of salivary acetaldehyde is 10-100 times higher than that in the blood. Acetaldehyde in smoking alcohol-dependent persons comes not only from the metabolism of ethanol but also from tobacco smoke. Besides acetaldehyde, tobacco smoke is a source of oxidative stress and contains up to 3000 different toxic substances such as nicotine, nitrosamines, carbon monoxide, and other aldehydes that may potentially damage the oral tissues $[22,23]$. In addition, ROS generated during drinking and smoking, as well as nonoxidative metabolites of ethanol (e.g., fatty acid ethyl esters, FAEEs) and the ethanol-water competition mechanism, might potentially be involved in the oral (periodontal) tissue damage $[8,12,22-$ 26]. It is generally known that chronic ethanol consumption leads to fat accumulation in salivary glands, acinar cell swelling, and atrophy and to reduction in weight and protein content of the parotid gland. Chronic drinking also reduces salivary flow rate, some of salivary proteins and glycoproteins levels (e.g., amylase), and sodium, bicarbonate, and chlorine concentrations $[12,27-30]$. A diffuse infiltration of salivary gland stroma by mononuclear inflammatory cells and their concentration around the salivary ducts were also reported [31]. Epithelial atrophy was also observed with a decrease in basal cellular size following chronic ethanol consumption. As compared to transient oral mucosa damage depending on the concentration of alcohol in acute alcohol ingestion, chronically intoxicated mucosa by the alcohol had dysplastic changes with the keratosis (atrophy with associated hyperregeneration) [21, 29]. In chronic cigarette smokers, swollen secretory cells of the salivary glands and decreased secretion of total salivary proteins were described $[26,32,33]$. Acetaldehyde from cigarette smoke has been shown to inactivate the most important antioxidant enzyme in the saliva-peroxidase [34]. Even in people chewing and snuffing tobacco, decreased levels of lysozyme and lactoferrin and increased secretion of immunoglobulin A in the saliva were found [33]. Although decreased secretion of total salivary proteins was described in smokers, significantly higher stimulated secretion of the saliva in smokers is due to the irritating effect of tobacco smoke on the oral mucosa [35]. In alcoholics who were active smokers, there were described decreased salivary flow and output of salivary acquired and innate immune proteins [8, $26,36,37]$.

Salivary enzymes, including exoglycosidases, come from gland cells, oral microorganisms, PMNs, and macrophages entering the gingival sulcus, as well as from epithelial cells, plasma, and dietary constituents. In particular, GCF outflow to the oral cavity is an important contributor to the enzyme pool in the whole saliva $[7,38]$. $\beta$-HEX is the most active of exoglycosidases that degrades tissue glycoconjugates including gingival tissue glycoconjugates. It was observed that the activity of $\beta$-HEX, which hydrolyses glycosidic linkages, decreased as a result of periodontitis treatment $[4,6]$. Therefore, $\beta$-HEX was suggested as a possible periodontitis marker. Another exoglycosidase, $\beta$-glucuronidase, that is a widely accepted periodontitis marker has a total predictive 
value that ranged from $83 \%$ to $88 \%$, with sensitivity ranged from $65 \%$ to $77 \%$ and specificity ranged from $85 \%$ to $89 \%$ [7].

Our study showed the significant increase in the salivary index of $\beta$-HEX A\% and decrease in $\beta$-HEX $\mathrm{B} \%$ in smoking alcoholics, as compared to the control group. As the activity of $\beta$-HEX $\mathrm{A}$ in body fluids is an inflammatory mediator, secreted into extracellular space by the inflammatory cells such as mast cells, basophils, macrophages, eosinophils, and neutrophils $[29,39,40]$, increased $\beta$-HEX A\% $(\beta$-HEX $\mathrm{A} /$ total $\beta$-HEX ratio) in our study seems to be due to the gingivitis. We found significant correlations between $\beta$-HEX A\% and GI. It is consistent with other studies in which the salivary $\beta$-HEX activities correlated with the degree of periodontal inflammation and decreased along with periodontal healing after treatment [4-6]. Increased $\beta$-HEX $\mathrm{A} \%$ ratio may also be due to the inflammatory state of the whole mucosa of the oral cavity and/or salivary glands and their subsequent hyperregeneration. A diffuse infiltration of the salivary gland stroma by mononuclear inflammatory cells and their concentration around the salivary ducts were earlier reported [31]. The periodontal inflammatory etiology of the high $\beta$-HEX A\% levels seems also to be confirmed by the significantly worse periodontal state in smoking alcoholics, reflected not only by higher periodontal inflammatory index, GI but also by higher papilla bleeding index, PBI, than in controls (Table 1).

The increased ratio of $\beta$-HEX A\% may also be due to the decrease in the salivary flow (SF) in alcoholics, as we found significant inverse correlations between $\mathrm{SF}$ and $\beta$ HEX A\%. Salivary flow (SF) was significantly lower than that in the control group, which confirms the literature data of chronic alcohol studies [27, 41]. Mostly, morphological changes of the salivary glands are considered to be involved in the SF reduction in people addicted to alcohol; other mechanisms involve risen liquidity of cell membranes, decreased secretory cell function, severe stress of the abstinence syndrome with accompanying sympathetic system arousal, hypercortisolemia, fluid diet and reduced chewing stimulation, decreased amount of muscarinic receptors, or deregulated secretion in the paracrine transmitters and gastrointestinal hormones, for example, cholecystokinin, somatostatin, and PP peptide $[12,26]$. A higher secretion of stimulated saliva in smokers is known $[12,35]$; hence, the SF reduction in alcohol-dependent smokers in our study seems to be rather the result of alcohol consumption than smoking.

Increased ratio of $\beta$-HEX A/total $\beta$-HEX $(\beta$-HEX A\%) indicates the increase in the $\beta$-HEX $\mathrm{A}$ isoenzyme activity, as shown in earlier study [12]. The increased $\beta$-HEX activity in the body fluids including saliva may occur in smoking alcoholics via many mechanisms, for example, increased lysosomal membrane permeability, delayed removal of the enzymes from the fluid, impaired trafficking of lysosomal hydrolases to organelles, enhanced synthesis of the enzyme by activated leucocytes, or leakage from damaged cells [12, 24, 25, 39]. Therefore, during periodontitis, the lysosomal and cellular membranes may be damaged, and proteases, together with glycosidases (including $\beta$-HEX), may be translocated into the cytosol fraction, the intercellular space, and to the body fluids (e.g., to the saliva) $[6,42,43]$.
Very high salivary levels of acetaldehyde derived from ethanol (10-100 times higher than in the blood) and cigarettes may be the reason of the lack of correlation of drinking or smoking with $\beta$-HEX $\mathrm{B} \%$ or $\beta$-HEX $\mathrm{A} \%$. Besides acetaldehyde, many other toxic substances such as nicotine, nitrosamines, carbon monoxide, and other aldehydes, [12, $22,23]$ in saliva of smoking alcohol-dependent persons may potentially be the reason of decreased ratio of $\beta$-HEX B\% due to $\beta$-HEX $\mathrm{B}$ inactivation. Although $\beta$-HEX $\mathrm{A}$ is a generally more labile isoenzyme than $\beta$-HEX $\mathrm{B}$ for high temperature, $\beta$-HEX B may be potentially more sensitive to highly concentrated acetaldehyde or other toxic compounds of the cigarette smoke. However, high correlation between GI and amount of smoked cigarettes per day may point to smoking as the main inductor of the bad gingival (periodontal) state.

The high value of the area under the curve (AUC) for $\beta$-HEX A\% index (0.937) in AS group, excellent sensitivity (96\%) and specificity (91\%) of $\beta$-HEX A\%, and its correlation with GI point to the high value of $\beta$-HEX A\% as an excellent periodontitis marker. Since a spectrophotometric method gives the same results as an immunoassay method to distinguish the activities of the $\beta$-HEX isoenzymes [17], the salivary $\beta$-HEX A\% determination may be a cheap and useful method for the detection of periodontitis in smoking alcoholics. Beta-HEX A\% index may be even a possible marker of periodontitis in a vast majority of the population, as we found correlations between $\beta$-HEX A\% and GI in smoking alcoholics together with controls. Although there are some limitations for our study, such as a lack of equal femalemale representation in $\mathrm{C}$ and $\mathrm{AS}$ groups and no group of periodontitis patients without history of alcohol and nicotine dependence, our study clearly shows that the salivary $\beta$-HEX $\mathrm{A} \%$ that correlates with gingival index has an excellent sensitivity and specificity. However, further studies need to check if there are similar high sensitivity and specificity of $\beta$-HEX $\mathrm{A} \%$ as a periodontitis marker in patients suffering from clear periodontitis (without substance dependence syndrome) and other pathological conditions.

As we noticed a tendency to the worse dental state (reflected by lower DMFT index) and significantly worse periodontal state (reflected by high GI and PBI indexes) (Table 1) in smoking alcoholics than in the controls, according to earlier studies, we confirmed the association between alcohol and nicotine addiction and poor dental state, periodontal state, and oral hygiene [12-15]. We also confirmed an earlier study [16] stating that cigarette smoking is a strong modifiable risk factor for periodontal disease. As we found a correlation of DMFT index and age, we confirmed data from the literature that the tooth loss is associated with increasing age [44]. It is also known that bad periodontal state worsens dental state [3], which we confirmed by a correlation of PBI and GI with DMFT index. Therefore, denture wearing seems to be a natural consequence of bad periodontal and subsequently bad dental states. We found high significant correlations between the time period of denture wearing and GI, PBI, and DMFT indexes. However, wearing of dentures is also a known major risk factor of periodontitis and caries, due to the poor oral hygiene, increased plaque and calculus accumulation, and transmission of excessive forces to the 
periodontal structures from occlusal surfaces of the framework of dentures $[45,46]$.

In conclusion, the increased salivary $\beta$-HEX $A \%$ index may be a possible excellent marker of the periodontitis. Smoking alcohol-dependent persons have worse periodontal state than controls, mostly due to the smoking-induced inflammatory state of the periodontium, as reflected by the high salivary $\beta$-HEX A\% index.

\section{Conflict of Interests}

The authors declare that they have no conflict of interests.

\section{References}

[1] S. N. Bhaskar, Orban's Oral Histology and Embryology, Mosby Year Book, St. Louis, Mo, USA, 13th edition, 2011.

[2] N. Buduneli and D. F. Kinane, "Host-derived diagnostic markers related to soft tissue destruction and bone degradation in periodontitis," Journal of Clinical Periodontology, vol. 38, supplement 11, pp. 85-105, 2011.

[3] N. Ozmeric, "Advances in periodontal disease markers," Clinica Chimica Acta, vol. 343, no. 1-2, pp. 1-16, 2004.

[4] G. J. van Steijn, A. V. Amerongen, E. C. Veerman, S. Kasanmoentalib, and B. Overdijk, "Effect of periodontal treatment on the activity of chitinase in whole saliva of periodontitis patients," Journal of Periodontal Research, vol. 37, no. 4, pp. 245-249, 2002.

[5] A. Nieminen, L. Nordlund, and V. J. Uitto, "The effect of treatment on the activity of salivary proteases and glycosidases in adults with advanced periodontitis," Journal of Periodontology, vol. 64, no. 4, pp. 297-301, 1993.

[6] R. Buchmann, A. Hasilik, M. E. Nunn, T. E. van Dyke, and D. E. Lange, "PMN responses in chronic periodontal disease: evaluation by gingival crevicular fluid enzymes and elastasealpha-1-proteinase inhibitor complex," Journal of Clinical Periodontology, vol. 29, no. 6, pp. 563-572, 2002.

[7] I. B. Lamster, L. G. Holmes, K. B. Gross et al., "The relationship of beta-glucuronidase activity in crevicular fluid to probing attachment loss in patients with adult periodontitis. Findings from a multicenter study," Journal of Clinical Periodontology, vol. 22, no. 1, pp. 36-44, 1995.

[8] N. Waszkiewicz, B. Zalewska-Szajda, A. Zalewska et al., "Decrease in salivary lactoferrin output in chronically intoxicated alcohol-dependent patients," Folia Histochemica et Cytobiologica, vol. 50, no. 2, pp. 248-254, 2012.

[9] World Health Organization, Global Status Report on Alcohol, Geneva, Switzerland, 2011.

[10] K. Ström, "Alcohol, smoking and lung disease," Addiction Biology, vol. 4, no. 1, pp. 17-22, 1999.

[11] D. J. Romberger and K. Grant, "Alcohol consumption and smoking status: the role of smoking cessation," Biomedicine and Pharmacotherapy, vol. 58, no. 2, pp. 77-83, 2004.

[12] N. Waszkiewicz, S. Chojnowska, A. Zalewska, K. Zwierz, A. Szulc, and S. D. Szajda, "Salivary hexosaminidase in smoking alcoholics with bad periodontal and dental states," Drug and Alcohol Dependence, vol. 129, pp. 33-40, 2013.

[13] N. Homann, J. Tillonen, H. Rintamäki, M. Salaspuro, C. Lindqvist, and J. H. Meurman, "Poor dental status increases acetaldehyde production from ethanol in saliva: a possible link to increased oral cancer risk among heavy drinkers," Oral Oncology, vol. 37, no. 2, pp. 153-158, 2001.
[14] B. L. Slomiany, Y. H. Liau, G. Zalesna, and A. Slomiany, "Effect of ethanol on the in vitro sulfation of salivary mucin," Alcoholism, vol. 12, no. 6, pp. 774-779, 1988.

[15] L. Hunter, R. Newcombe, S. Richmond, J. Owens, and M. Addy, "The Cardiff dental survey: oral hygiene, gingival and periodontal health in relation to smoking in young adults," International Journal of Dental Hygiene, vol. 6, no. 3, pp. 199-204, 2008.

[16] G. K. Johnson and J. M. Guthmiller, "The impact of cigarette smoking on periodontal disease and treatment," Periodontology 2000, vol. 44, no. 1, pp. 178-194, 2007.

[17] L. Stowell, A. Stowell, N. Garrett, and G. Robinson, "Comparison of serum $\beta$-hexosaminidase isoenzyme $B$ activity with serum carbohydrate-deficient transferrin and other markers of alcohol abuse," Alcohol and Alcoholism, vol. 32, no. 6, pp. 703714, 1997.

[18] C. Dawes, "Physiological factors affecting salivary flow rate, oral sugar clearance, and the sensation of dry mouth in man," Journal of Dental Research, vol. 66, pp. 648-653, 1987.

[19] M. Navazesh, C. Christensen, and V. Brightman, "Clinical criteria for the diagnosis of salivary gland hypofunction," Journal of Dental Research, vol. 71, no. 7, pp. 1363-1369, 1992.

[20] J. Marciniak, A. Zalewska, J. Popko, and K. Zwierz, "Optimization of an enzymatic method for the determination of lysosomal $\mathrm{N}$-acetyl- $\beta$-D-hexosaminidase and $\beta$-glucuronidase in synovial fluid," Clinical Chemistry and Laboratory Medicine, vol. 44, no. 8, pp. 933-937, 2006.

[21] N. Waszkiewicz, S. D. Szajda, A. Jankowska et al., “The effect of acute ethanol intoxication on salivary proteins of innate and adaptive immunity," Alcoholism: Clinical and Experimental Research, vol. 32, no. 4, pp. 652-656, 2008.

[22] N. Waszkiewicz, A. Zalewska, A. Szulc et al., "The influence of alcohol on the oral cavity, salivary glands and saliva," Polski Merkuriusz Lekarski, vol. 30, no. 175, pp. 69-74, 2011.

[23] M. I. Ryder, "The influence of smoking on host responses in periodontal infections," Periodontology 2000, vol. 43, no. 1, pp. 267-277, 2007.

[24] N. Waszkiewicz, S. D. Szajda, A. Kępka, A. Szulc, and K. Zwierz, "Glycoconjugates in the detection of alcohol abuse," Biochemical Society Transactions, vol. 39, no. 1, pp. 365-369, 2011.

[25] N. Waszkiewicz, S. D. Szajda, A. Zalewska et al., "Alcohol abuse and glycoconjugate metabolism," Folia Histochemica et Cytobiologica, vol. 50, no. 1, pp. 1-11, 2012.

[26] N. Waszkiewicz, A. Zalewska, S. D. Szajda et al., "The effect of chronic alcohol intoxication and smoking on the activity of oral peroxidase," Folia Histochemica et Cytobiologica, vol. 50, no. 3, pp. 450-455, 2012.

[27] S. K. Dutta, M. Orestes, S. Vengulekur, and P. Kwo, "Ethanol and human saliva: effect of chronic alcoholism on flow rate, composition, and epidermal growth factor," The American Journal of Gastroenterology, vol. 87, no. 3, pp. 350-354, 1992.

[28] B. L. Slomiany, J. Piotrowski, and A. Slomiany, "Chronic alcohol ingestion enhances tumor necrosis factor- $\alpha$ expression and salivary gland apoptosis," Alcoholism, vol. 21, no. 8, pp. 1530 1533, 1997.

[29] F. Riedel, U. Goessler, and K. Hörmann, "Alcohol-related diseases of the mouth and throat," Bailliere's Best Practice and Research in Clinical Gastroenterology, vol. 17, no. 4, pp. 543-555, 2003.

[30] J. K. M. Aps and L. C. Martens, "Review: the physiology of saliva and transfer of drugs into saliva," Forensic Science International, vol. 150, no. 2, pp. 119-131, 2005. 
[31] P. F. Smith, J. C. Ramirez, R. E. Romo, and A. S. Esguep, "Salivary glands in alcoholism: a histologic and sialographic study," Addiction Biology, vol. 1, no. 4, pp. 385-393, 1996.

[32] H. Maier, I. A. Born, and G. Mall, "Effect of chronic ethanol and nicotine consumption on the function and morphology of the salivary glands," Klinische Wochenschrift, vol. 66, supplement 11, pp. 140-150, 1988.

[33] J. D. Rudney, "Does variability in salivary protein concentrations influence oral microbial ecology and oral health?" Critical Reviews in Oral Biology and Medicine, vol. 6, no. 4, pp. 343-367, 1995.

[34] A. Z. Reznick, I. Klein, J. P. Eiserich, C. E. Cross, and R. M. Nagler, "Inhibition of oral peroxidase activity by cigarette smoke: in vivo and in vitro studies," Free Radical Biology and Medicine, vol. 34, no. 3, pp. 377-384, 2003.

[35] V. D. A. Pdel, A. M. Gregio, M. A. Machado, A. A. de Lima, and L. R. Azevedo, "Saliva composition and functions: a comprehensive review," Journal of Contemporary Dental Practice, vol. 9, no. 3, pp. 72-80, 2008.

[36] N. Waszkiewicz, A. Zalewska, S. D. Szajda et al., "The effect of chronic alcohol intoxication and smoking on the output of salivary immunoglobulin A," Folia Histochemica et Cytobiologica, vol. 50, pp. 605-608, 2012.

[37] N. Waszkiewicz, B. Zalewska-Szajda, A. Zalewska et al., "Salivary lysozyme in smoking alcohol-dependent persons," Folia Histochemica et Cytobiologica, vol. 50, pp. 609-612, 2012.

[38] N. Waszkiewicz, S. D. Szajda, A. Jankowska et al., "Catabolism of salivary glycoconjugates in acute ethanol intoxication," Medical Science Monitor, vol. 15, no. 8, pp. CR413-CR417, 2009.

[39] N. Waszkiewicz, S. D. Szajda, A. Jankowska et al., "The effect of the binge drinking session on the activity of salivary, serum and urinary $\beta$-hexosaminidase: preliminary data," Alcohol and Alcoholism, vol. 43, no. 4, pp. 446-450, 2008.

[40] D. B. Lew, B. K. Dempsey, Y. Zhao, M. Muthalif, S. Fatima, and K. U. Malik, " $\beta$-Hexosaminidase-induced activation of p $44 / 42$ mitogen-activated protein kinase is dependent on p21Ras and protein kinase $\mathrm{C}$ and mediates bovine airway smooth-muscle proliferation," American Journal of Respiratory Cell and Molecular Biology, vol. 21, no. 1, pp. 111-118, 1999.

[41] H. Maier, I. A. Born, S. Veith, D. Adler, and H. K. Seitz, "The effect of chronic ethanol consumption on salivary gland morphology and function in the rat," Alcoholism, vol. 10, no. 4, pp. 425-427, 1986.

[42] N. Waszkiewicz, S. D. Szajda, A. Zalewska et al., "Binge drinking-induced liver injury," Hepatology, vol. 50, no. 5, p. 1676, 2009.

[43] N. Waszkiewicz, R. Popławska, B. Konarzewska et al., "Biomarkers of alcohol abuse-Part II: new biomarkers and their interpretation," Psychiatria Polska, vol. 44, no. 1, pp. 137-146, 2010.

[44] J. G. Steele, A. E. Sanders, G. D. Slade et al., "How do age and tooth loss affect oral health impacts and quality of life? A study comparing two national samples," Community Dentistry and Oral Epidemiology, vol. 32, no. 2, pp. 107-114, 2004.

[45] E. Budtz-Jörgensen, "Effects of denture-wearing habits on periodontal health of abutment teeth in patients with overdentures," Journal of Clinical Periodontology, vol. 21, no. 4, pp. 265-269, 1994.

[46] D. K. Zlatarić, A. Čelebić, and M. Valentić-Peruzović, "The effect of removable partial dentures on periodontal health of abutment and non-abuntment teeth," Journal of Periodontology, vol. 73, no. 2, pp. 137-144, 2002. 


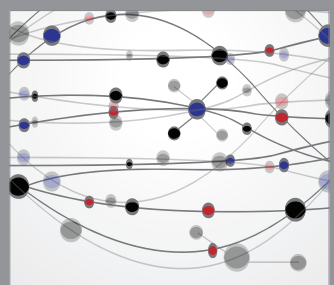

The Scientific World Journal
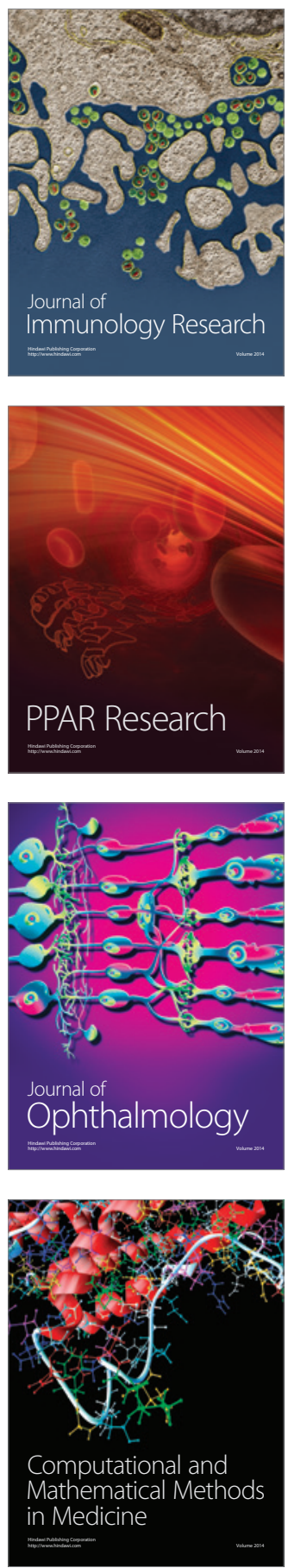

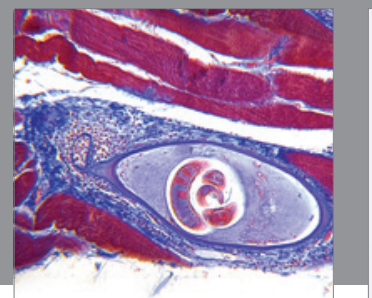

Gastroenterology

Research and Practice
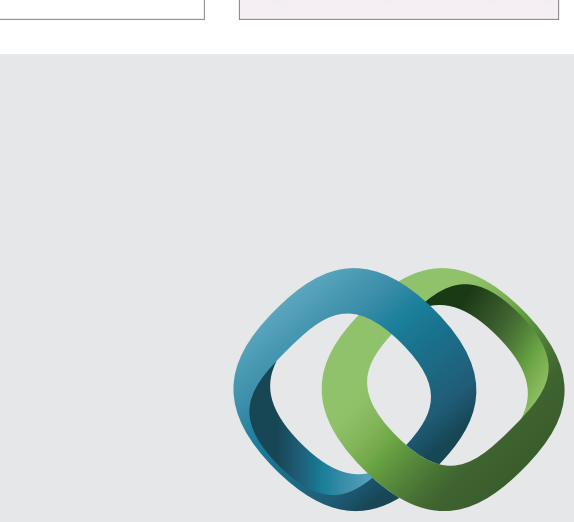

\section{Hindawi}

Submit your manuscripts at

http://www.hindawi.com
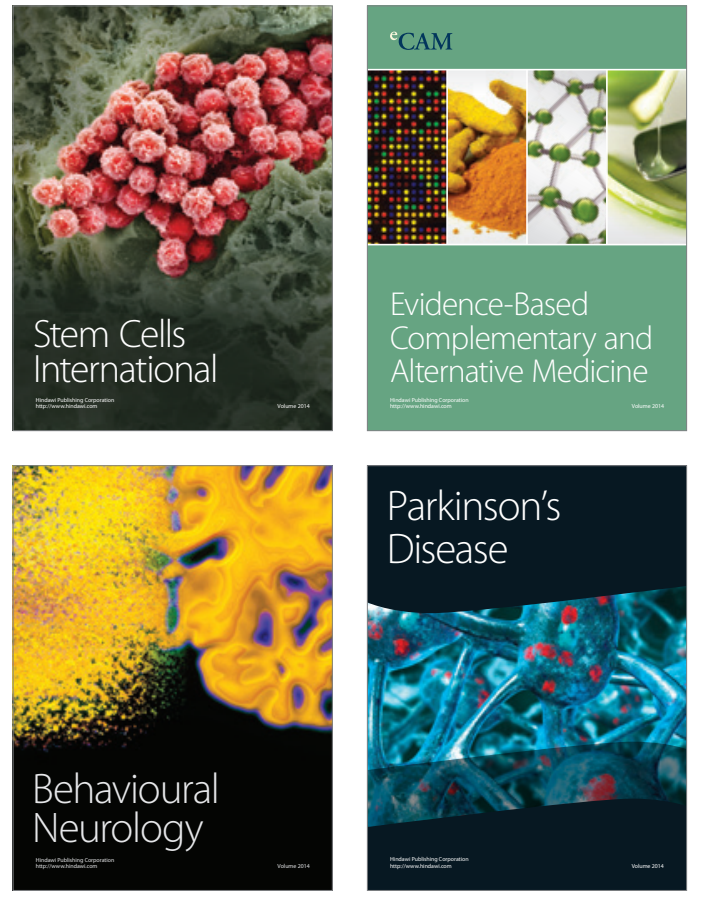
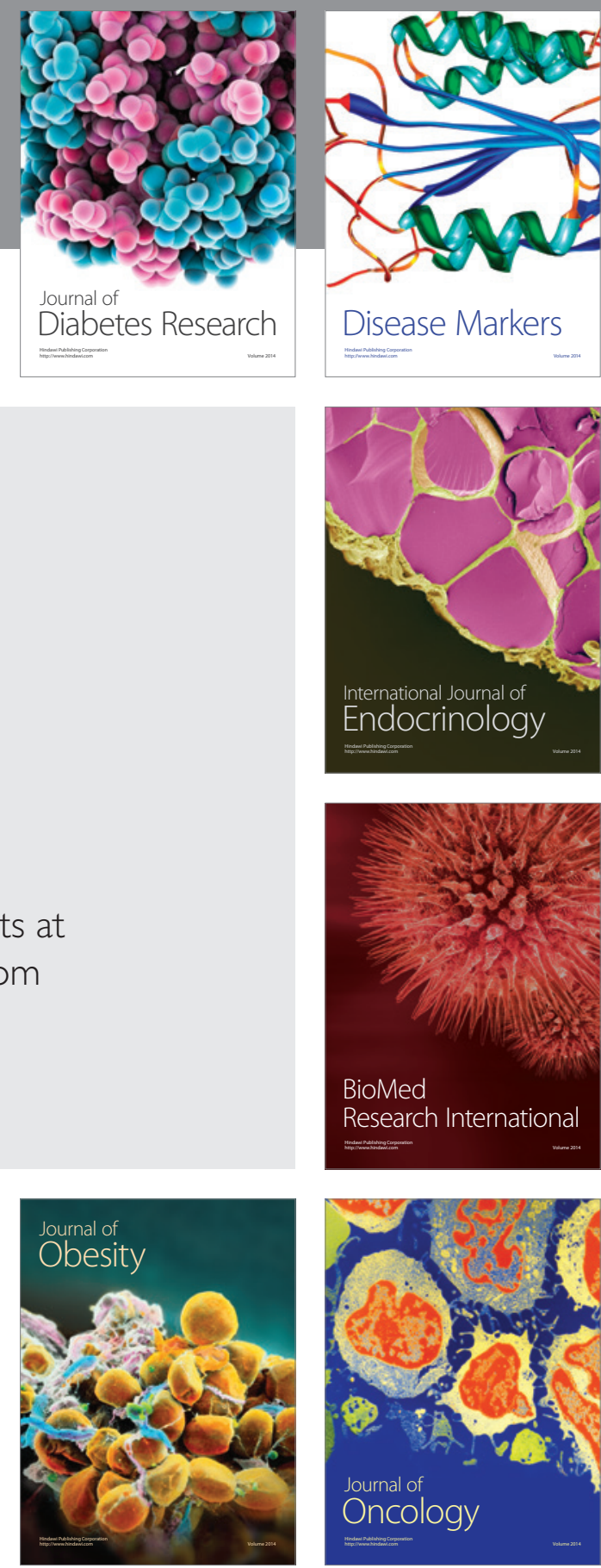

Disease Markers
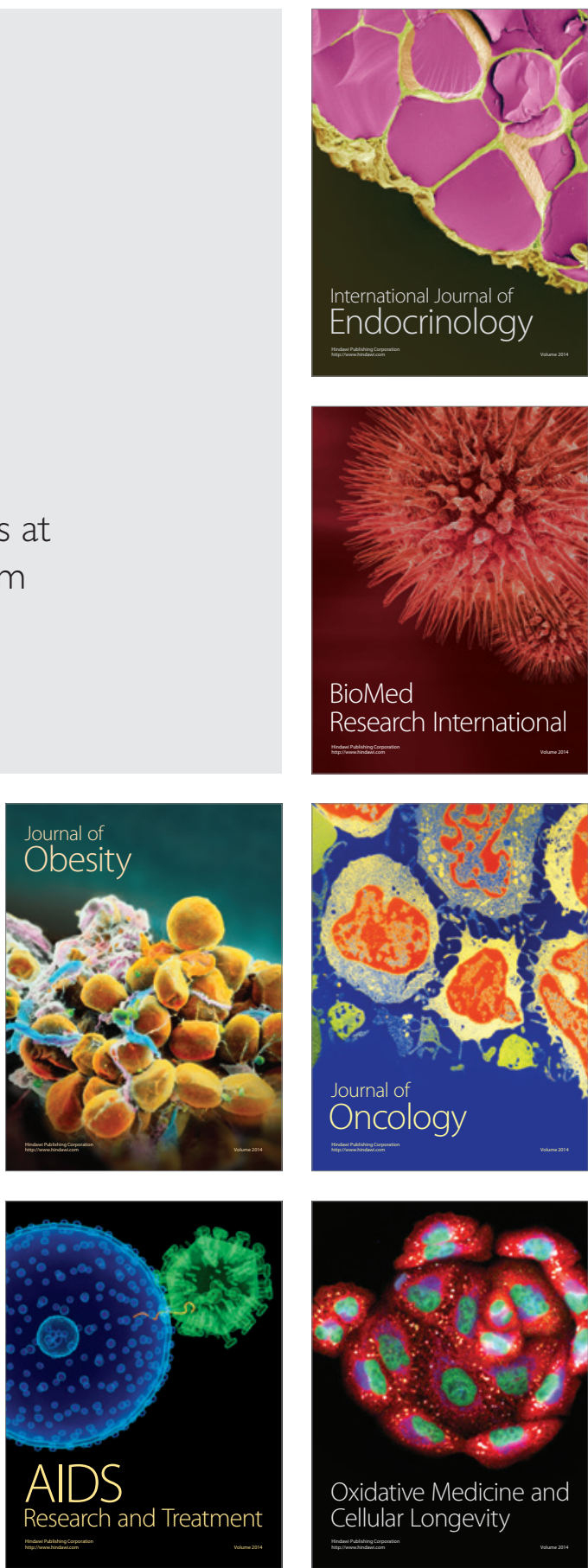